Draft Version August 5, 2021

Preprint typeset using $\mathrm{LAT}_{\mathrm{E}} \mathrm{X}$ style emulateapj v. 6/22/04

\title{
HIGH-RESOLUTION INFRARED IMAGING OF HERSCHEL 36 SE: A SHOWCASE FOR THE INFLUENCE OF MASSIVE STARS IN CLUSTER ENVIRONMENTS ${ }^{1,2}$
}

\author{
M. Goto,${ }^{3}$ B. Stecklum,${ }^{4}$ H. Linz,${ }^{3,4}$ M. Feldt,${ }^{3}$ Th. Henning, ${ }^{3}$ I. Pascucci, ${ }^{3,5}$, T. Usuda ${ }^{6}$ \\ Draft version August 5, 2021
}

\begin{abstract}
We present high-resolution infrared imaging of the massive star-forming region around the O-star Herschel 36. Special emphasis is given to a compact infrared source at 0.25 southeast of the star. The infrared source, hereafter Her $36 \mathrm{SE}$, is extended in the broad-band images, but features spatially unresolved $\operatorname{Br} \gamma$ line emission. The line-emission source coincides in position with the previous HST detections in $\mathrm{H} \alpha$ and the $2 \mathrm{~cm}$ radio continuum emission detected by $V L A$ interferometry. We propose that the infrared source Her 36 SE harbors an early B-type star, deeply embedded in a dusty cloud. The fan shape of the cloud with Herschel 36 at its apex, though, manifests direct and ongoing destructive influence of the O7V star on Her 36 SE.

Subject headings: circumstellar matter — dust, extinction — planetary systems: protoplanetary disks — early-type — stars: formation — stars: individual (Herschel 36, G5.97-1.17)
\end{abstract}

\section{INTRODUCTION}

Massive stars are the primary source of radiation, kinetic energy, and chemical enrichment in the interstellar medium, playing a pivotal role in galactic evolution. Because of their remote locations, our understanding of their formation has been limited by the lack of high resolution techniques. This challenge has been undertaken by adaptive optics systems at large-aperture telescopes. The present work is part of a coordinated effort to understand the formation of high-mass stars by using state-of-the-art instruments available at the $V L T$ and other telescopes (Feldt et al. 1999; Henning et al. 2001; Feldt et al. 2003; Gradv et al. 2004; Pascucci et al. 2004; Puga et al. 2004; Linz et al. 2005; Apai et al.|2005).

Herschel 36 is located in a high-mass star-forming region at a distance of $1.8 \mathrm{kpc}$ from us (van den Anker et al. 1997; but see also Arias et al. 2006) near the center of M 8. The bright central part of M 8 is called the Hourglass. The Hourglass is a cavity of ionized gas seen through the gaps between the foreground obscuration (Woodward et al. 1986). Herschel 36, an O7V star (Woolf 1961), is responsible for the ionization of the gas in the cavity. The inferred dynamical age of the ionized gas and, therefore, the age of the Hourglass and Herschel 36 , is as small as $\sim 5 \times 10^{4}$ yrs (Chakrabortv \& Anandarao 1997).

In the present study a special focus is placed on the infrared source found at a distance of 0.25 southeast of Herschel 36. The extended source called hereafter

\footnotetext{
Electronic address: mgoto@mpia-hd.mpg.de

1 Based on data collected in the course of NACO and MIDI guaranteed time observations (71.C-0143(A) and 73.C-0175(A)) at the VLT on Cerro Paranal (Chile), which is operated by the European Southern Observatory (ESO).

2 Based on data collected at Subaru Telescope, which is operated by the National Astronomical Observatory of Japan.

3 Max Planck Institute for Astronomy, Königstuhl 17, D-69117 Heidelberg, Germany.

4 Thüringer Landessternwarte Tautenburg, Sternwarte 5, D07778 Tautenburg, Germany.

5 The University of Arizona, Department of Astronomy/Steward Observatory, 993 N Cherry Ave. Tucson, AZ 85721-0065.

6 Subaru Telescope, 650, North A'ohoku Place, Hilo, HI 96720.
}

Her 36 SE was first recognized by Stecklum et al. (1995) by means of lunar occultation measurements. Herschel 36 had long been known as a peculiar early-type star with substantial mid-infrared excess (Woolf 1973; Allen 1986). It is only after Her $36 \mathrm{SE}$ was spatially resolved that we know that this object is actually the source responsible for the excess infrared emission. After the discovery of Stecklum et al. (1995), the possible identity of Her 36 SE has been discussed, including an externally ionized protoplanetary disk, or, a proplyd, an obscured embedded source, and a leftover circumstellar disk of Herschel 36; however, no solid conclusion was reached.

In the next section, the observations at the $V L T$ and supplemental spectroscopy at the Subaru Telescope are described. The direct consequences of the observations are summarized in $\S 3$. In $\S 4$ we will further discuss the possible nature of Her $36 \mathrm{SE}$ as a deeply embedded earlytype star under the violent influence of the nearby O-star Herschel 36 .

\section{OBSERVATION AND DATA REDUCTION}

\subsection{Thermal Near-infrared Imaging}

The infrared imaging at $L^{\prime}(3.8 \mu \mathrm{m})$ and $M^{\prime}(4.7 \mu \mathrm{m})$ was carried out at the VLT UT4 on 2003 June 11 with the adaptive optics imager NACO (Rousset et al. 2000; Lenzen et al. 2003; Hartung et al. 2003). Herschel 36 $(V=9.1 \mathrm{mag})$ served as a wavefront reference source for the visible wavefront sensor in the adaptive optics system. A short exposure of $180 \mathrm{~ms}$ was repeated 27 times in the $L^{\prime}$ imaging at each of the 9 positions of the telescope dithering. Imaging at $M^{\prime}$ was performed the same way, but with a shorter integration time of $56 \mathrm{~ms}$ repeated 89 times. The total on-source integration time is $58 \mathrm{~s}$ and $60 \mathrm{~s}$ at $L^{\prime}$ and $M^{\prime}$, respectively. The observing $\log$ is presented in Table 1 including imaging with other filters and additional spectroscopy.

The imaging data were reduced in the standard manner. After sky-subtraction and flat-fielding, images were registered referring to the position of Herschel 36 . The size of the isoplanatic patch was measured using more than 40 stars inside the entire field of view of 


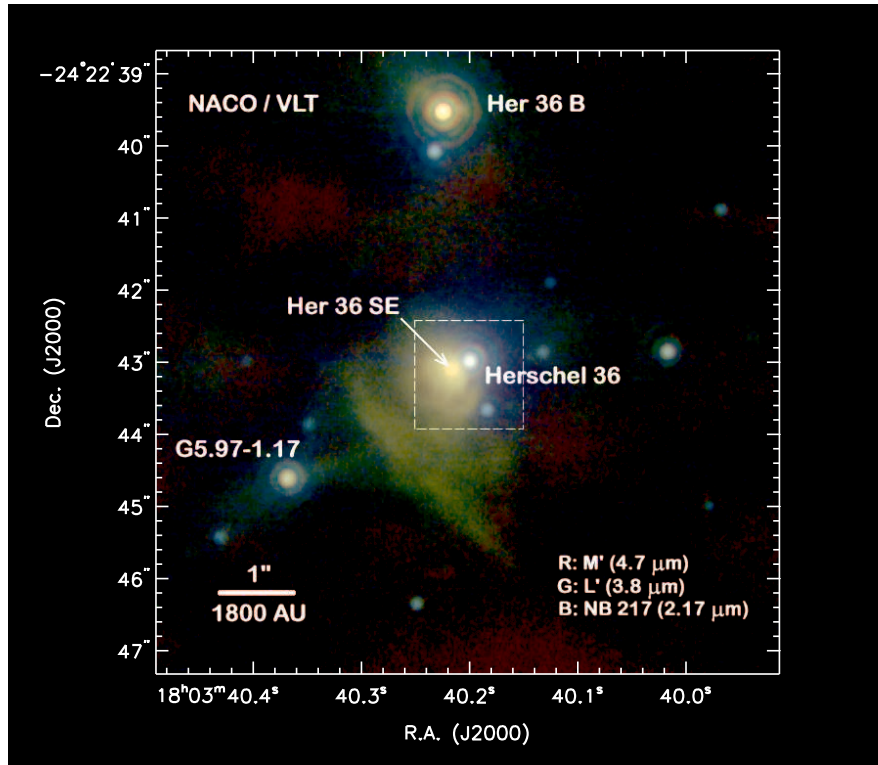

FIG. 1.- Color composite image of Herschel 36. $M^{\prime}$ is color coded in red, $L^{\prime}$ in green, and $\operatorname{Br} \gamma$ in blue before continuum subtraction. Her $36 \mathrm{SE}$ is the red extended emission at $00^{\prime \prime} 25$ southeast of Herschel 36. Her $36 \mathrm{~B}$ is an infrared star at 3.'6 north of Herschel 36. The coordinates of Herschel 36 are R.A. (J2000) $18^{\mathrm{h}} 03^{\mathrm{m}} 40.20$, Dec (J2000) $-24^{\circ} 22^{\prime} 43^{\prime \prime} 0$ (Maiz-Apellaniz et al. 2004).

NACO $\left(27^{\prime \prime} \times 27^{\prime \prime}\right)$. The measurements have been done at $2.2 \mu \mathrm{m}$, since the isoplanatic patch becomes smaller with the wavelength. The point spread function (PSF) is found elongated only at the edge of the field of view, and no significant degradation of PSF is recognized within the field of view relevant to the following discussion shown in Figure 11 The full width at half maximum (FWHM) of point sources are $0^{\prime \prime} .11$ at $L^{\prime}$ and $0^{\prime \prime} .13$ at $M^{\prime}$ in the fully reduced images.

The absolute flux calibration was tied to the photometry of Herschel 36 as given in the literature. The zero point magnitudes were calculated to be consistent with the photometric magnitudes $L^{\prime}=6.3 \mathrm{mag}$ and $M^{\prime}=$ $3.8 \mathrm{mag}$ in Woodward et al. (1990) and Woolf (1973), respectively. The images were convolved with Gaussian filter at the zero-point calculation to match the spatial resolution in the previous observations. The photometry of Herschel 36 was performed inside a small aperture of 0 '! 2 to avoid confusion with Her $36 \mathrm{SE}$. An aperture correction was applied by using the PSF sampled at Her $36 \mathrm{~B}$ (see Figure 1). The photometry of Her $36 \mathrm{SE}$ was then performed in a circular aperture of 1 !! 3 , after the contribution of Herschel 36 was removed by subtracting the scaled PSF. The primary error source in the photometry is the spatial fluctuation of the background emission in the immediate vicinity of Herschel 36 . We restored the flux compensation function with varying outer bounds from 0.6 to 1 .' 4 , and found that the amount of aperture correction does not differ more than $15 \%$. The sky level sampled at different locations at 0.9 to 2 .' 6 around the object does not change the net photometry by more than $14 \%$. We therefore quote $0.2 \mathrm{mag}$ as the photometric accuracy, although the formal error is much smaller $(<0.05 \mathrm{mag})$. The results are presented in Table 2 with other photometry obtained in this paper. The color com- posite image of $L^{\prime}, M^{\prime}$ and the narrow-band image at $2.17 \mu \mathrm{m}$ described in the next section is shown in Figure 1

\subsection{Narrow-band Imaging at $B r \gamma$}

Narrow-band $\operatorname{Br} \gamma$ images $(2.17 \mu \mathrm{m})$ were obtained during the same night together with continuum images at $2.18 \mu \mathrm{m}$. Short exposures of $1 \mathrm{~s}$ were repeated 20 times to minimize the saturation of bright stars. The total integration time on source is $100 \mathrm{~s}$. The sky at $30^{\prime \prime}$ distance from the field was recorded for background subtraction. The data were processed in the same way as it was the case for broadband imaging. Aperture photometry was performed for Her $36 \mathrm{SE}$ in $\mathrm{Br} \gamma$ and the continuum at $2.18 \mu \mathrm{m}$. Since Herschel 36 is saturated, Her 36 B was used to establish the correct flux scale of the images $(K=9.4 \mathrm{mag}$; KS1 in Woodward et al. 1990, 18006nr766 in Bik 2004). The PSF sampled from Her $36 \mathrm{~B}$ was scaled and subtracted from Herschel 36 to isolate the extended emission of Her $36 \mathrm{SE}$. The total pixel counts were summed up inside a circular aperture of $1^{\prime \prime} .3$ centered on Her 36 SE. Note that the $\mathrm{Br} \gamma$ photometry presented in Table 2 is before the continuum subtraction. The accuracy of the photometry is $\sim 0.1 \mathrm{mag}$ for the images with the underlying continuum emission. The continuum image was scaled and subtracted so that the pixel counts of blue stars (with respect to their infrared colors) around Herschel 36 are equally canceled in the line-emission image.

\subsection{Mid-infrared Imaging at $8.7 \mu \mathrm{m}$ and $12.8 \mu \mathrm{m}$}
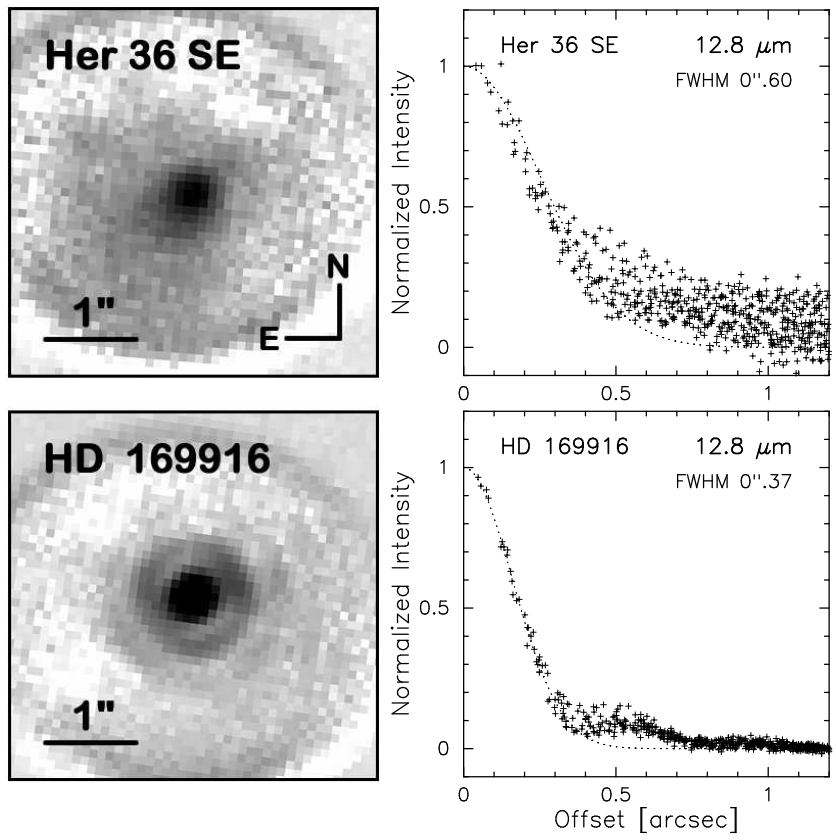

FIG. 2.- Left: MIDI source-acquisition images at $12.8 \mu \mathrm{m}$ for Her 36 SE (top) and the flux calibration star HD 169916 (bottom). Herschel 36 is no longer visible at this wavelength. While HD 169916 has multiple diffraction rings around, Her 36 SE is extended with no trace of a point source. Right: The radial profile of Her $36 \mathrm{SE}$ and HD 169916. The size of the emitting region at Her $36 \mathrm{SE}$ is $850 \mathrm{AU}$ in diameter, after deconvolution with the spatial profile of HD 169916. 
TABLE 1

\begin{tabular}{|c|c|c|c|c|c|c|c|}
\hline UT Date & Filter/Band & $\lambda[\mu \mathrm{m}]$ & $\Delta \lambda^{\mathrm{a}}[\mu \mathrm{m}]$ & Telescope & Instrument & Observation & Spectral Resolution \\
\hline 2003 Jun 11 & $L^{\prime}$ & 3.80 & 0.62 & $V L T$ & $\mathrm{NACO}$ & Imaging & \\
\hline 2003 Jun 11 & $M^{\prime}$ & 4.78 & 0.59 & $V L T$ & NACO & Imaging & \\
\hline 2003 Jun 11 & $\mathrm{Br}, \gamma$ & 2.17 & 0.023 & $V L T$ & $\mathrm{NACO}$ & Imaging & \\
\hline 2003 Jun 11 & IB 218 & 2.18 & 0.060 & $V L T$ & $\mathrm{NACO}$ & Imaging & \\
\hline 2004 Jun 3 & N8.7 & 8.64 & 1.55 & $V L T$ & MIDI & Imaging & \\
\hline 2004 Jun 3 & {$[\mathrm{Ne} \mathrm{II}]$} & 12.8 & 0.39 & $V L T$ & MIDI & Imaging & \\
\hline 2004 Jul 29 & $\operatorname{Br} \alpha$ & 4.05 & & Subaru & IRCS/AO & Spectroscopy & $R=10,000$ \\
\hline
\end{tabular}

${ }^{\mathrm{a}}$ In FWHM.

Mid-infrared images in the $N 1$ filter $(8.7 \mu \mathrm{m})$ and in the $[\mathrm{Ne}$ II] filter $(12.8 \mu \mathrm{m})$ were obtained on 2004 June 3 at the VLT with MIDI (Leinert et al. 2003). The instrument is an interferometer/spectrometer, but was used as mid-infrared camera in the present observation. The tiptilt corrector STRAP was used to stabilize the images. These thermal infrared data were recorded by using a chopping throw of $10^{\prime \prime}$. The total on-source integration is $80 \mathrm{~s}$ at $8.7 \mu \mathrm{m}$ and $375 \mathrm{~s}$ at $12.8 \mu \mathrm{m}$. The data reduction was carried out using the pipeline provided by the MIDI consortium ${ }^{7}$. The spatial resolution of the final image is nearly diffraction limited (Fig. 2).

The emission from Her $36 \mathrm{SE}$ is clearly extended both in $N 1$ and [Ne II]. Herschel 36 is no longer visible at mid-infrared wavelengths, as is expected from its photospheric spectral energy distribution (SED). It is clear that the peculiar mid-infrared excess toward Herschel 36 (Dvck 1977) is not from the O-star itself, but is almost entirely attributed to Her $36 \mathrm{SE}$. The flux calibration was performed with respect to the photometric standard HD 169916 for which the absolute flux density was taken from Cohen et al. (1999). The photometry was performed inside a $1^{\prime \prime} .8$ aperture centered on Her 36 SE. The size of the aperture is slightly larger than that is used in the shorter wavelengths. The smaller aperture at the thermal near-infrared is because there seem to be two overlapping emission contributions at Her 36 SE; the compact dusty cloud at Her 36 SE itself, and the filamentary emission more connected to the diffuse emission at $2^{\prime \prime}$ southeast of Herschel 36 . Since we will discuss an internal source inside Her 36 SE below, the diffuse emission should be excluded not to overestimate its luminosity. On the other hand, the mid-infrared images by MIDI do not show any clear hints of multiple sources, we therefore use a safe oversized aperture not to lose the mid-infrared flux of Her 36 SE for later discussion of its energetics.

\subsection{Medium-Resolution Br $\alpha$ Spectroscopy}

Supplemental 4-micron spectroscopy $(R=10,000)$ was performed at the Subaru Telescope on 29 July 2004 with IRCS (Tokunaga et al. 1998; Kobavashi et al. 2000). The slit was oriented along a position angle of $110^{\circ}$ counted from north to east to cover Herschel 36 and $\mathrm{SE}$ at the same time. An adaptive optics system was used to attain higher spatial resolution (Gaessler et al. 2002; Takami et al. 2004). The sky at $2^{\prime}$ north of Herschel 36 was observed for background subtraction after

\footnotetext{
${ }^{7}$ http://www.mpia-hd.mpg.de/MIDISOFT/
}

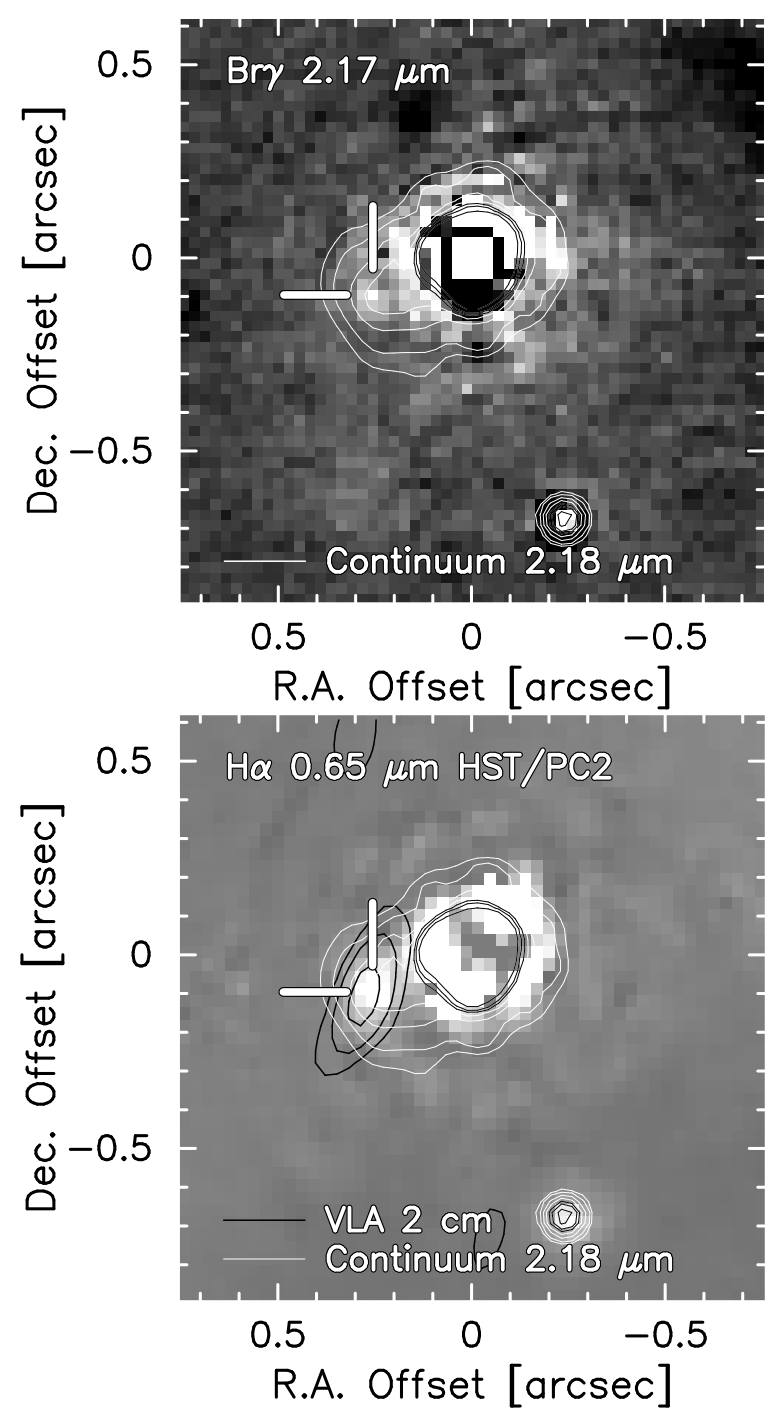

FIG. 3.- (a) Continuum-subtracted $\mathrm{Br} \gamma$ image of Her 36 SE. The location of the point source is marked by ticks. The gray contour is from the narrow-band continuum image at $2.18 \mu \mathrm{m}$ presented darker at around Herschel 36 for clarity. No point source is detected at the continuum wavelength. (b) The $\mathrm{H} \alpha$ image retrieved from the HST archive. The PSF generated by TinyTim is subtracted. The contour in black is radio continuum emission at $2 \mathrm{~cm}$ obtained with the VLA (see Stecklum et al. 1998 for observational detail). The unresolved sources at $\operatorname{Br} \gamma, \mathrm{H} \alpha$, and radio wavelengths are all in positional agreement. The coordinate offsets are with respect to Herschel 36.

each on-source integration. The spectroscopic flat field 

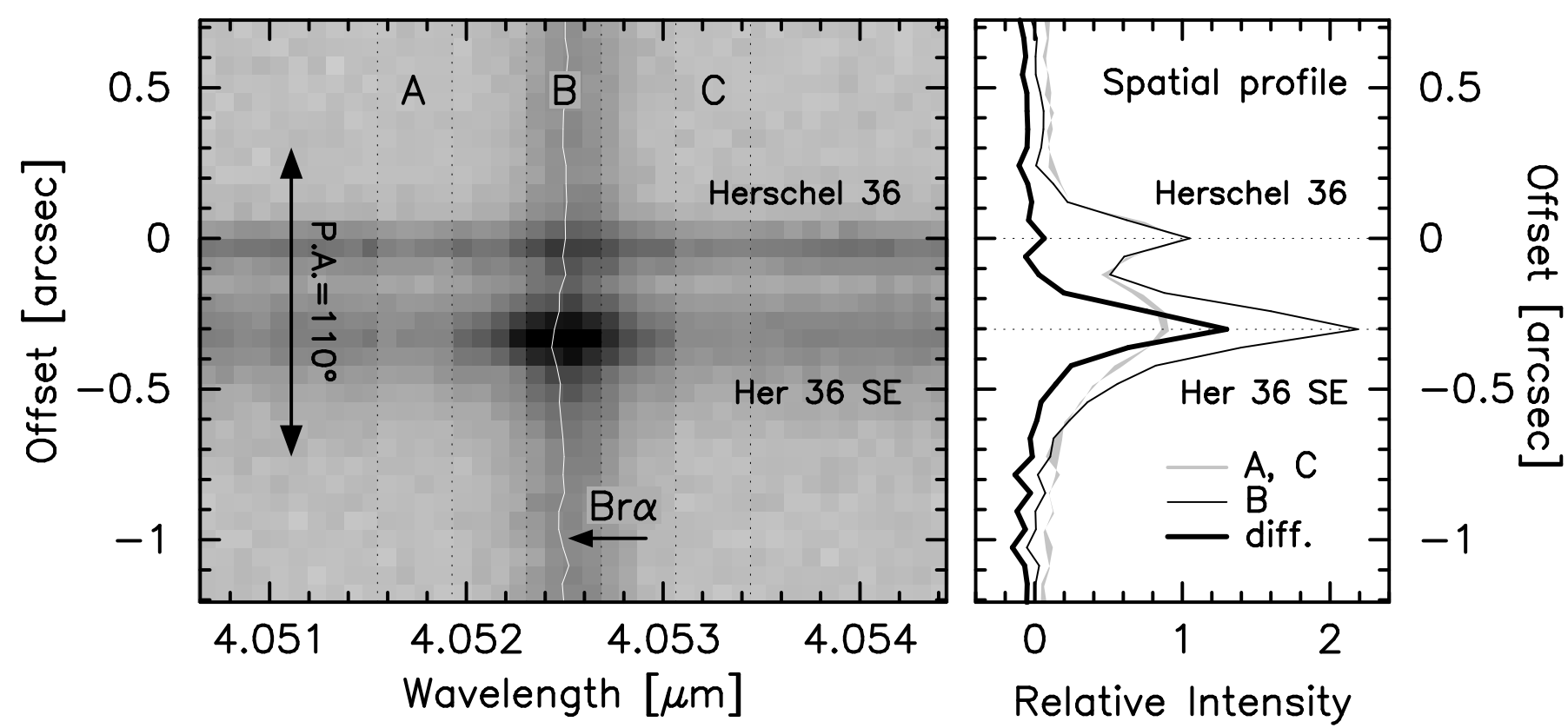

FIG. 4.- Left: A close-up view of the two-dimensional spectrogram near the Br $\alpha$ emission. The continuum emission at Her 36 SE is clearly separated from that of Herschel 36, and apparently extended (position A and C). The line emission from the ambient nebulosity is seen at $\operatorname{Br} \alpha$ (position B). The white line traces the wavelength centroids of the $\operatorname{Br} \alpha$ emission along the slit. The emission line is slightly $\left(\sim 2 \mathrm{~km} \mathrm{~s}^{-1}\right)$ blueshifted at Her $36 \mathrm{SE}$ with respect to the ambient nebular emission. Right: Cross-cuts of the spectrogram along the slit, at the continuum (A and $\mathrm{C}$; gray polygon), and the $\operatorname{Br} \alpha$ emission (B; thin solid line). The contribution of the nebulosity is subtracted at the position B. Her 36 SE shows a sharp emission line at $\operatorname{Br} \alpha$ on top of the extended continuum emission. The line emission profile, after subtracting the continuum profile, is close to that of Herschel 36, which means that the Br $\alpha$ emission at Her 36 SE is also compact and point-like.

was obtained from a halogen lamp exposure at the end of the night.

One dimensional spectra of Herschel 36 and SE were extracted with the aperture-extraction package of IRAF ${ }^{8}$ after the sky-subtraction, flat-fielding, and the interpolation of the bad pixels were applied. The wavelength calibration was carried out using the atmospheric transmission curve modeled by ATRAN (Lord 1992). The $\operatorname{Br} \alpha$ line-emission was calibrated to the photometry of Herschel 36 at $L^{\prime}$. First, to correct the continuum slope, the one-dimensional spectrum of Herschel 36 was divided by the spectroscopic standard star HR 7121 (B2.5V), and was multiplied by a blackbody spectrum of the temperature corresponding to the effective temperature of a B2.5V star $\left(T_{\text {eff }}=19,000 \mathrm{~K}\right.$; Crowther 2005$)$. The spectrum with correct slope was then scaled so that the averaged flux density inside the $L^{\prime}$ bandpath $(3.49-4.11 \mu \mathrm{m})$ is equal to the $L^{\prime}$-band photometry of Herschel 36 . The same conversion factor was used to calibrate the spectral flux of Her 36 SE reduced in the same way with Herschel 36. The $\operatorname{Br} \alpha$ luminosity at Her $36 \mathrm{SE}$ is found to be $L(\operatorname{Br} \alpha)=(1.4-1.6) \times 10^{24} \mathrm{~W}$ at the distance of $1.8 \mathrm{kpc}$, after the continuum and the surrounding diffuse emission is subtracted. The error interval is given by the difference of the two sequential measurements, although it is subject to the uncounted uncertainty associated to the possible vignetting by the narrow slit of 0.3 .

\section{RESULTS}

8 IRAF is distributed by the National Optical Astronomy Observatories, which are operated by the Association of Universities for Research in Astronomy, Inc., under cooperative agreement with the National Science Foundation.
An unresolved source is detected at the location of Her $36 \mathrm{SE}$ in the continuum-subtracted image at $\mathrm{Br} \gamma$ (Fig. 31). The diameter of the point-like source is less than $130 \mathrm{AU}$ from the diffraction-limited spatial resolution of NACO ( $0^{\prime \prime} 072$ in FWHM at $\left.2.17 \mu \mathrm{m}\right)$. The $H S T / \mathrm{PC} 2$ image retrieved from the $\mathrm{ST}-\mathrm{ECF}^{9}$ archive shows a compact $\mathrm{H} \alpha$ emission at the same location (Fig. 3). The $\mathrm{H} \alpha$ emission is unresolved as well. Considering the plate scale of PC2 $\left(00^{\prime \prime} 046\right.$ pixel $\left.^{-1}\right)$, this finding indicates that the source is less than 100 AU across. Furthermore, radio interferometric observations have been carried out with the VLA at a wavelength of $2 \mathrm{~cm}$ (see Stecklum et al. 1998 for the observational detail). A compact radio source is found at the same location as the position of the $\operatorname{Br} \gamma$ and $\mathrm{H} \alpha$ emission. The radio source is unresolved with regard to the synthesized beam size of 0 !' 16 , which translates to 290 AU.

Another line of evidence for a point-like source comes from the spectroscopy. The two-dimensional spectrogram near $\operatorname{Br} \alpha$ is shown in Figure 4 . Her $36 \mathrm{SE}$ shows distinct line emission in $\operatorname{Br} \alpha$, slightly blueshifted from ambient nebular emission by $2 \mathrm{~km} \mathrm{~s}^{-1}$, with a spatial profile apparently narrower than the continuum emission. The sharp spatial profile is comparable to that of Herschel 36, which corroborates the presence of a point-like source in the hydrogen line emission at the location of Her 36 SE.

On the other hand, Her $36 \mathrm{SE}$ is clearly extended in

9 The Space Telescope European Coordinating Facility (STECF), jointly operated by ESA and the European Southern Observatory (ESO), is the European HST science facility, supporting the European astronomy community in exploiting the research opportunities provided by the Hubble Space Telescope. 
the continuum emission at wavelengths from 2 to $13 \mu \mathrm{m}$. If we use the spatial profile of HD 169916 as the instrumental PSF, and deconvolve Her 36 SE by inverting simple square sum, the extent of the emitting source at Her $36 \mathrm{SE}$ is reduced to $0 . ! 47$ at $12.8 \mu \mathrm{m}$, which is $850 \mathrm{AU}$ in diameter at the distance of the object (Fig. 2). The SED of Her $36 \mathrm{SE}$ is presented in Figure 5 to characterize the extended emission. The color temperature of the continuum source clearly points to the existence of warm dust at the location of Her 36 SE. No point-like substructure is found in the broad-band images of Her 36 SE that could have corresponded to the unresolved line emission.

\section{DISCUSSION - NATURE OF HERSCHEL 36 SE}

Here we first discuss the identity of the line emission source and its possible ionization mechanism, including external ionization by Herschel 36, an embedded low- to intermediate-mass star in its active accretion phase, and an H II region internally ionized by an early-type star.

The $\operatorname{Br} \gamma$ emission is apparently inside the dusty cloud at Her $36 \mathrm{SE}$, since it is spatially more confined than the continuum emission. In addition, there is no hint of rim-ionization detected in $\operatorname{Br} \gamma$ emission at the side of Her 36 SE where it faces toward Herschel 36. We found no solid evidence that Herschel 36 plays a direct role to externally ionize the unresolved source inside Her 36 SE.

The radio flux at $2 \mathrm{~cm}\left(F_{\nu}=1.3 \mathrm{mJy}\right)$ is probably too high for an accretion signature of an intermediate-mass star at $1.8 \mathrm{kpc}$ away. Neufeld \& Hollenbach (1996) have calculated the free-free emission arising from an accretion shock in dependence of (proto)stellar mass and accretion rate. However, even with their most extreme setup ( $M$ $\left.=10 M_{\odot}, \dot{M}_{\text {acc }}=10^{-4} M_{\odot} \mathrm{yr}^{-1}\right)$ they just reach a $3.6 \mathrm{~cm}$ flux of roughly $3 \mathrm{mJy}$ for a source $100 \mathrm{pc}$ away. Extrapolated to $\lambda=2 \mathrm{~cm}$ (by optimistically assuming that the ionized gas is completely optically thick with $F_{\nu} \sim \nu^{2}$ ) and scaled to a distance of $1.8 \mathrm{kpc}$, the expected $2-\mathrm{cm}$ flux would be just some $30 \mu \mathrm{Jy}$, around 40 times less than the measured value.

It is therefore inferred that a star with an early spectral type exists inside Her $36 \mathrm{SE}$ that gives rise to a small H II

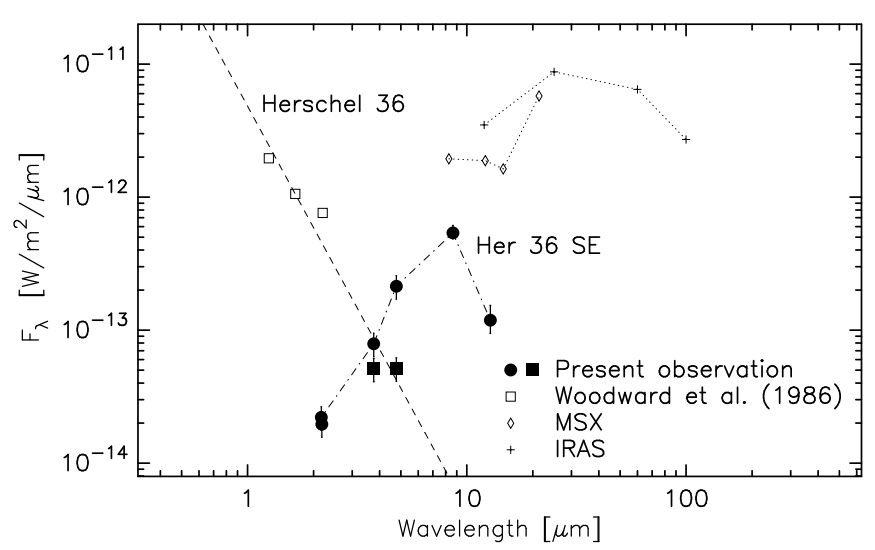

FIG. 5. - The infrared SED of Herschel 36 and SE, plotted by the circles and the squares, respectively. The filled symbols are from the present observations, and the crosses and open symbols are from the literature. Note that this previous photometry has been done with much larger apertures. The color temperature of the dust emission measures $400 \mathrm{~K}$ from $3-13 \mu \mathrm{m}$ photometry, which is taken as the upper limit of the dust emission temperature of Her 36 SE. region responsible for the radio emission. The $\mathrm{H}$ II region is internally ionized, but is kept compact because of the high density of the enshrouding cloud. If we take the $V L A$ beam size as the physical dimension of the $\mathrm{H}$ II region $(r \sim 140 \mathrm{AU})$, the number of Lyman continuum photons required to maintain the ionized gas is $1.6 \times 10^{45}$ $\mathrm{s}^{-1}$. The Lyman continuum photon-rate is reproducible only by a star earlier than B2 if the star is at the zeroage main sequence (Panagia 1973; Crowther 2005). On the other hand, in order not to create a parsec-scale H II region, the hydrogen density has to be as high as $n_{\mathrm{H}}>$ $10^{6} \mathrm{~cm}^{-3}$.

We use the $K$-band extinction toward the hypothetical early-type star to estimate whether the gas density is sufficiently high to confine the H II region. A B2 star at the zero-age main-sequence should have a relative $K$ band brightness of $9.9 \mathrm{mag}$ at the distance of Herschel 36 without any attenuation $\left((V-K)_{0}=-0.9 \mathrm{mag}\right.$ from Ducati et al. 2001). The sensitivity of our observation at $2 \mu \mathrm{m}$ is $15.7 \mathrm{mag}$ for a 3 sigma detection at the location of Her 36 SE. With non-detection of any continuum point source at this wavelength, the dust extinction must be larger than $6 \mathrm{mag}$ at $2 \mu \mathrm{m}$, which translates to $A_{V}>$ $60 \mathrm{mag}$ after correcting $A_{V} \approx 5 \mathrm{mag}$ for the foreground extinction toward the Herschel 36 region (Stecklum et al. 1998). The visible extinction can be related to a hydrogen column density (e.g., Mathis 1990; Rvter 1996). Provided that the dusty core of Her $36 \mathrm{SE}$ is spherical, of constant density, and $850 \mathrm{AU}$ across as is measured in the $12.8 \mu \mathrm{m}$ image; the mass in the obscuration is $M_{\mathrm{SE}} \geq 1.7 \times 10^{-2} M_{\odot}$ with $n_{\mathrm{H}} \geq 1.8 \times 10^{7} \mathrm{~cm}^{-3}$. Thus, the gas density should be high enough to keep the H II region spatially unresolved.

The Lyman photon rate derived from $\operatorname{Br} \alpha$ spectroscopy is also consistent with that of an early B type star. The ionizing flux in an H II region is obtained from $\operatorname{Br} \alpha$ line flux by scaling the photon numbercount proportionally to the recombination coefficients, $\int_{\nu_{0}} L_{\nu} / h \nu d \nu \approx \alpha_{B} / \alpha_{\mathrm{Br} \alpha}^{\mathrm{eff}} \cdot L(\operatorname{Br} \alpha) / h \nu_{\mathrm{Br} \alpha}$. If we take $\alpha_{\mathrm{Br} \alpha}^{\mathrm{eff}}=1.085 \times 10^{-14} \mathrm{~cm}^{3} \mathrm{~s}^{-1}$ and $\alpha_{B}=2.658 \times$ $10^{-13} \mathrm{~cm}^{3} \mathrm{~s}^{-1}$ from Storev \& Hummer (1995) for the Case B of $T_{\mathrm{e}}=10^{4} \mathrm{~K}$ and $N_{\mathrm{e}}=10^{7} \mathrm{~cm}^{-3}$, the Lyman continuum rate turns out $(6.9-8.0) \times 10^{44} \mathrm{~s}^{-1}$, which is reproducible by a B2.5 dwarf (Crowther 2005). The correction of the foreground extinction needs caution, since the dust obscuration is increasingly transparent in the longer wavelength (Rieke \& Lebofskv 1985); but if we use $A_{K}=6 \mathrm{mag}$ as a face value, the intrinsic Lyman continuum rate is $(4.1-4.8) \times 10^{45} \mathrm{~s}^{-1}$, which is still consistent with the ionizing photon rate of a B1-B1.5 dwarf.

The infrared luminosity of Her $36 \mathrm{SE}$ is consistent both with an internal B2 star, and also with external heating by Herschel 36. The infrared luminosity from 2 to $40 \mu \mathrm{m}$ is calculated from the NACO and MIDI photometry with the flux density at the longer wavelength extrapolated as $F(\lambda)=\kappa(\lambda) M_{d} d^{-2} B\left(T_{d}, \lambda\right)$; where $d$ is the distance to the object, and $\kappa(\lambda)$ is the computed mass absorption coefficient for the grains without ice mantles coagulated in the protostellar cores (Ossenkopf \& Henning 1994). The total infrared luminosity is $L_{\mathrm{IR}}=400 L_{\odot}$ at an assumed distance of $1.8 \mathrm{kpc}$ insensitive to the gas density of the core $n=10^{6} \mathrm{~cm}^{-3}$ to $10^{8} \mathrm{~cm}^{-3}$. It is therefore well reproducible either by the luminosity of a B2 star at the 
TABLE 2

\begin{tabular}{|c|c|c|c|}
\hline & Herschel 36 & Her $36 \mathrm{SE}^{\mathrm{a}}$ & \\
\hline Filter & $F_{\lambda}{ }^{\mathrm{b}}\left[10^{-14} \mathrm{~W} \mathrm{~m}^{-2} \mu \mathrm{m}^{-1}\right]$ & $F_{\lambda}{ }^{\mathrm{b}}\left[10^{-14} \mathrm{~W} \mathrm{~m}^{-2} \mu \mathrm{m}^{-1}\right]$ & PSF in FWHM \\
\hline $\operatorname{Br} \gamma$ & $\cdots$ & $2.2^{\mathrm{c}}$ & $0 .{ }^{\prime \prime} 072$ \\
\hline IB 218 & $\cdots$ & 2.0 & $0 . \prime 071$ \\
\hline$L^{\prime}$ & 5.1 & 7.9 & $0 !$ \\
\hline$M^{\prime}$ & 5.2 & 21 & $0 !$ \\
\hline N8.7 & $\cdots$ & 54 & $0 . \prime 35$ \\
\hline $\mathrm{Ne}$ II & $\cdots$ & 12 & $0 . \prime 37$ \\
\hline
\end{tabular}

aThe photometric apertures are $1 . ! 3$ in the mid-infrared bands (N8.7 and Ne II), and 1.' 8 in all the others.

b The photometric errors are typically $10 \%$ at $2.2 \mu \mathrm{m}$, and $20 \%$ at all the other filters.

${ }^{\mathrm{c}}$ Continuum is not subtracted. zero-age main-sequence $\left(L_{*}=3 \times 10^{3} L_{\odot}\right)$, or by Herschel $36\left(L_{*}=10^{5} L_{\odot}\right)$ while the solid angle subtended by Her $36 \mathrm{SE}$ is of the order of unity at the location of Herschel 36. The dust emitting temperature is $400 \mathrm{~K}$ which should be taken as the upper limit, for the lack of additional photometry at the longer wavelengths.

We conclude by comparing Her $36 \mathrm{SE}$ with two similar cases reported to date in which bright mid-infrared sources are found in the immediate (projected) vicinity of O-type stars. The infrared source SC3 has been found at 1 .' $8(810 \mathrm{AU})$ west of $\theta^{1}$ Ori $\mathrm{C}$, the primary illumination source of the Orion Trapezium Cluster (Havward, Houch, \& Miles 1994). SC3 is spatially resolved, measuring 1..5 across, however, despite the apparent proximity to $\theta^{1}$ Ori C $(\mathrm{O} 5.5 \mathrm{~V})$, its appearance is barely distorted, almost with a perfect circular symmetry. It is thus proposed that SC3 is a proplyd seen face-on, located deep behind $\theta^{1}$ Ori $\mathrm{C}$ with the physical separation much larger than the apparent projection (Robberto. Beckwith. \& Panagia 2002). SC3 is visible in the optical (e.g., Smith et al. 2005), and at near-infrared wavelengths (McCaughrean \& Stauffer 1994), which also lends support to its proplyd nature. The infrared appearance of SC3 is in strong contrast to Her 36 SE. We may use the highly distorted dust emission from Her 36 SE as circumstantial evidence that the source is actually under the influence of Herschel 36, and that the physical distance to the $\mathrm{O}$ star is not significantly larger than it appears.

On the other hand, $\sigma$ Ori IRS 1 , found next to $\sigma$ Orionis, shares a similar morphology with Her $36 \mathrm{SE}$. It is a compact infrared source at 1200 AU away from the O9.5V star, and features a fan-shaped emission with $\sigma$ Orionis at the apex (van Loon \& Oliveira 2003), exactly as Herschel 36 is to SE. The mid-infrared spectrum of $\sigma$ Ori IRS 1 shows the partly crystalline silicate in emission. The presence of processed silicates suggests significant grain growth which is naturally present in a circumstellar disk. A proplyd is therefore again the most probable cause of $\sigma$ Ori IRS 1, especially because a central star has been detected recently in the $K$-band continuum emission (B. Stecklum, private communication).

The star-forming region around Herschel 36 has many features in common with the Orion Nebula Cluster. The local concentration of massive stars, like Her 36 B, together forms a Trapezium-like cluster around Herschel 36. The presence of known proplyd nearby at G5.97-1.17 (Stecklum et al. 1998) underscores the physical similarity as well. The mid-infrared color of Her $36 \mathrm{SE}$, and the close vicinity to an O-type star with a distorted morphology suggestive of radiative influence of it all point toward Her $36 \mathrm{SE}$ is also a proplyd with a low-mass star at its center, as is the case for SC3 at $\theta^{1}$ Ori $\mathrm{C}$ and $\sigma$ Ori IRS 1 . However, in addition to the radio luminosity hardly accounted for by a low-mass star, and no ionized-front outside the dusty cloud; a proplyd cannot explain the lack of a point source to be detected at the continuum wavelengths that comes from the photospheric emission of the star. In the case of no central star inside, another possibility would be that Her $36 \mathrm{SE}$ is an evaporating gaseous globule, a failed proplyd without an internal star in formation. These starless cores have been detected in numbers toward M 16 (McCaughrean \& Andersen 2002). However, a hypothetical starless globule conflicts with the presence of the unresolved $\operatorname{Br} \gamma$ emission apparently inside of Her 36 SE. We therefore propose that Her 36 SE harbors a relatively massive star of early B-type producing a squeezed H II region inside the dusty cloud (Keto 2003), but the star itself is completely obscured. The distortion of dust emission as well as the diffuse emission downstream of Her 36 SE, indicates the close physical interaction of Herschel 36 and SE. Herschel $36 \mathrm{SE}$, now in the process of being blown away, is a showcase for the violent impact of the dominant O-star in a cluster on another early-type star nearby.

We thank all the staff and crew of the VLT and Subaru for their valuable assistance in obtaining the data, and Thorsten Ratzka, Elena Puga and Wolfgang Brandner for their indispensable help in reducing data. We appreciate the anonymous referee for many critical comments that are necessary to improve the paper. M.G. is supported by Japan Society for the Promotion of Science fellowship.

\section{REFERENCES}

Arias, J. I., Barbá, R. H., Maíz Apellánz, J., Morrell, N. I., \& Rubio, M. 2006, MNRAS, 366, 739
Apai, D., Linz, H., Henning, Th., \& Stecklum, B. 2005, A\&A, 434, 987 
Allen, D. A. 1986, MNRAS, 219, 35

Bik, A. 2004, PhD thesis, Universiteit van Amsterdam

Charkraborty, A., \& Anandarao, B. G. 1997, AJ, 114, 1576

Cohen, M., et al. 1999, AJ, 117, 1864

Crowther, P. A., Massive Star Birth: A Crossroads of Astrophysics, International Astronomical Union. Symposium no. 227, 389 on May 2005 in Italy, eds. R. Cesaroni, M. Felli, E. Churchwell. M. Walmsley, Cambridge: Cambridge University Press, astro-ph/0506324

Ducati, J. K., Bevilacqua, C. M., Rembold, S. B., \& Ribeiro, D. 2001, ApJ, 558, 309

Dyck, H. M. 1977, AJ, 1977, 82, 129

Feldt, M., Stecklum, B., Henning, Th., Launhardt, R., \& Hayward, T. L. 1999, A\&A, 346, 243

Feldt, M., Puga, E., Lenzen, R., Henning, Th., Brandner, W., Stecklum, B., Lagrange, A.-M., Gendron, E., \& Rousset, G. 2003, ApJ, 599, L91

Gaessler, W., et al. 2002, Proc. SPIE, 4494, 30

Grady, C. A., Woodgate, B., Torres, Carlos A. O., Henning, Th. Apai, D., Rodmann, J., Wang, Hongchi, Stecklum, B., Linz, H., Williger, G. M., Brown, A., Wilkinson, E., Harper, G. M., Herczeg, G. J., Danks, A., Vieira, G. L., Malumuth, E., Collins, N. R., \& Hill, R. S. 2004, ApJ, 608, 809

Hartung, M., Lenzen, R., Hofmann, R., Böhm, A., Brandner, W., Finger, G., Fusco, T., Lacombe, F. Laun, W., Ganier, P., Storz, C., \& Wagner, K. 2003, Proc. SPIE, 4841, 425

Hayward, T. L, Houch, J. R., \& Miles, J. W. 1994, ApJ, 433, 157

Henning, Th., Feldt, M., Stecklum, B., \& Klein, R. A\&A, 2001, 370,100

Kobayashi, N., et al. 2000, Proc. SPIE, 4008, 1056

Keto, E. 2003, ApJ, 599, 1196

Leinert, C., Graser, U., Waters, L. B. F. M., Perrin, G. S., Walter J., Bruno, L., Przygodda, F., Chesneau, O., Schuller, P. A., Grlazenborg-Kluttig, Annelie, W., Laun, W., Ligori, S., Meisner, J. A., Wagner, K., Bakker, E. J., Cotton, B.; de Jong, J., Mathar, R., Neumann, U., \& Storz, C. 2003, Proc. SPIE 4838, 893

Lenzen, R., Hartung, M., Brandner, W., Finger, G., Hubin, N. N., Lacombe, F., Lagrange, A.-M., Lehnert, M. D., Moorwood, A. F. M., \& Mouillet, D. 2003, Proc. SPIE, 4841, 860

Linz, H., Stecklum, B., Henning, Th., Hofner, P., \& Brandl, B. 2005, A\&A, 429, 903

Lord, S. D. 1992, A New Software Tool for Computing Earth's Atmosphere Transmissions of Near- and Far-Infrared Radiation, NASA Technical Memoir 103957 (Moffett Field, CA: NASA Ames Research Center)
Maiz-Apellaniz, J., Walborn, N. R., Galue H. A., Wei, L. H. 2004, ApJS, 151, 103

Mathis, J. S. 1990, ARA\&A, 28, 37

McCaughrean, M. J., \& Stauffer, J. R. 1994, AJ, 108, 1382

McCaughrean, M. J., \& Andersen, M. 2002, A\&A, 389, 513

Neufeld, D. A., \& Hollenbach, D. J. 1996, ApJ, 471, L45

Ossenkopf, V., \& Henning, Th. 1994, A\&A, 291, 943

Panagia, N. 1973, AJ, 78929

Pascucci, I., Apai, D., Henning, Th., Stecklum, B., \& Brandl, B. 2004, A\&A, 426, 523

Puga, E., Alvarez, C., Feldt, M., Henning, Th., \& Wolf, S. 2004, A\&A, 425, 543

Rieke, G. H., \& Lebofsky, M. J. 1985, ApJ, 288, 618

Robberto, M., Beckwith, S. V. W., \& Panagia, N. 2002, ApJ, 578, 897

Rousset, G., Lacombe, F., Puget, P., Gendron, E., Arsenault, R., Kern, P. Y., Rabaud, D., Madec, P.-Y., Hubin, N. N., Zins, G., Stadler, E., Charton, J., Gigan, P., Feautrier, P. 2000, Proc. SPIE, 407, 72

Ryter, Ch. E. 1996, Ap\&SS, 236, 285

Smith, N., Bally, J., Shuping, R. Y., Morris, M., \& Kassis, M. 2005, AJ, 130,1778

Stecklum, B., Henning, Th., Eckart, A., Howell, R. R., \& Hoare, M. G. 1995, ApJ, 445, L153

Stecklum, B., Henning, Th., Feldt, M., Hayward, T. L, Hoare, M. G., Hofner, P., \& Richter, S. 1998, AJ, 115, 767

Storey, P. J., \& Hummer, D. G. 1995, MNRAS

Takami, H., et al. 2004, PASJ, 56, 225

Tokunaga, A. T., et al. 1998, Proc. SPIE, 3354, 512

van Loon, J. Th., \& Oliveira, J. M. 2003, A\&A, 405, L33

van den Ancker, M. E., Thé, P. S., Feinstein, A., Vázquez, R. A., de Winter, D., \& Pérez, M. R. 1997, A\&AS, 123, 63

Woodward, C. E., Pipher, J. L., Helfer, H. L., Sharpless, S., Moneti, A., Kozikowski, D., Oliveri, M., Willner, S. P., Lacasse, M. G., \& Herter, T. 1986, AJ, 91, 870

Woodward, C. E., Pipher, J. L, Helfer, H. L, \& Forrest, W. J. 1990, ApJ, 365, 252

Woolf, N. J. 1961, PASP, 73, 206

Woolf, N. J., Stein, W. A., Gillett, F. C., Merrill, K. M., Becklin, E. E., Neugebauer, G., Pepin, T. J., 1973, ApJ, 179, L111 